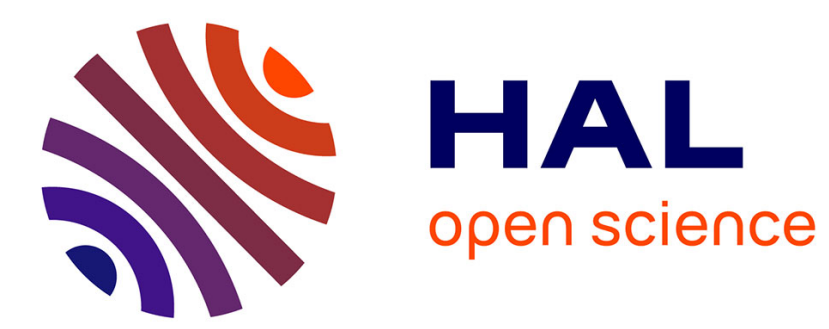

\title{
DISCUSSION PANEL
}

P. Kessler, F. Martin, D. Lyth, K. Subrarao

\section{To cite this version:}

P. Kessler, F. Martin, D. Lyth, K. Subrarao. DISCUSSION PANEL. Journal de Physique Colloques, 1974, 35 (C2), pp.C2-119-C2-120. 10.1051/jphyscol:1974217 . jpa-00215527

\section{HAL Id: jpa-00215527 https://hal.science/jpa-00215527}

Submitted on 1 Jan 1974

HAL is a multi-disciplinary open access archive for the deposit and dissemination of scientific research documents, whether they are published or not. The documents may come from teaching and research institutions in France or abroad, or from public or private research centers.
L'archive ouverte pluridisciplinaire HAL, est destinée au dépôt et à la diffusion de documents scientifiques de niveau recherche, publiés ou non, émanant des établissements d'enseignement et de recherche français ou étrangers, des laboratoires publics ou privés. 


\title{
DISCUSSION PANEL
}

\author{
P. KESSLER, F. MARTIN, D. H. LYTH and K. SUBBARAO
}

B. LAUTRUP. - I have a question to Dr. Subbarao : Does the Moller interference term, which you have neglected, not influence the $L^{0}$ term ?

K. Subbarao. - Yes, actually, it should affect the $M_{3}$ term. I have not calculated its contribution.

R. MAdaras (CEA). - For the proposed SPEAR experiment which will tag the scattered electrons at angles of about $5^{\circ}$, how good is the $W W$ approximation in the calculation of the cross section?

P. Kessler (Paris). - The condition we fixed for the validity of the $W W$ approximation is $:\left|q^{2}\right| \ll W^{2}$, i. e. $E \theta \ll W$. lt thus depends not only on energy and angle of the electrons, but also on the invariant mass created. For instance, for $E \approx 3 \mathrm{GeV}, W \approx 500 \mathrm{MeV}$, the condition would be : $\theta \ll 10^{\circ}$. However, I must stress that, when you go to large angles (more than a few degrees), the main problem is not the breakdown of the $W W$ approximation, but - as Parisi has shown yesterday - the background due to the competing diagrams.

D. H. LYTH (Lancaster). - In our work, we defined the conditions : $\left|q^{2}\right| \ll M_{\mathrm{H}}^{2}$, where $M_{\mathrm{H}}$ is the smallest relevant hadron mass; and $\left|q^{2}\right| \ll \omega^{2}$, where $\omega$ is the virtual photon's energy.

P. Kessler. - Actually, Lyth and Brown's conditions are a bit stricter than ours, so that we disagree perhaps to some extent with them on this technical detail. In practice, however, I think this does not make a big difference.

H. Terazawa (Rockefeller University). - I strongly recommend that experimentalists should detect scattered electrons within a small angle of the order of a few degrees, so that they can use the modified equivalent photon approximation formula. Since then this formula can be taken as exact, the question on the validity of the approximation would disappear.

P. Kessler. - This statement is indeed confirmed by the remarkable curves shown in the paper by Brown and Lyth (Nucl. Phys. B 53 (1973) 323), where it can be seen that, for a cut-off angle of a few degrees imposed on the electrons (on both sides) in

$$
\text { ee } \rightarrow \mathrm{ee} \pi^{+} \pi^{-},
$$

there is practically no difference, as to the integrated cross section, between the $W W$ approximation used by them and the exact calculation.

G. Salvini (Rome). - To detect the electrons at small angles is the real challenge for the experimentalists in the next years. We must notice that the invariant hadronic mass will not be known in general. So the limits underlined by Kessler and Terazawa are even more severe.

P. Kessler. - Yes, in that case the limit should actually be $:\left|q^{2}\right| \ll W_{\min }^{2}$, i. e. the transfer should be kept small with respect to the threshold mass.

F. MARTIN (Paris). - I want to make a remark on Terazawa's statement. We noticed that all the equivalent photon methods agree for

$$
q^{2} \ll W^{4} /(4 s) \simeq 3 \times 10^{-6} \mathrm{GeV}^{2}
$$

(for $W=m_{\pi^{0}}$ and beam energy $3 \mathrm{GeV}$ ); such $q^{2}$ values correspond to very small limit angles $\left(\simeq 6 \times 10^{-4} \mathrm{rad}\right)$. Above that limit, the different versions of the equivalent photon approximation can differ by 20 to $30 \%$.

N. Cabibbo (Rome). - The idea of «equivalent photons" has further applications: one can use «cascade approximations". As an example, an electron can be equivalent to an electron plus an electronpositron pair, through the following cascade :

$$
\mathrm{e}^{ \pm} \rightarrow \mathrm{e}^{ \pm}+\gamma, \quad \gamma \rightarrow \mathrm{e}^{+}+\mathrm{e}^{-} .
$$

This approximation was used to compute the class B contribution to the process

$\mathrm{e}^{+}+\mathrm{e}^{-} \rightarrow\left(\mathrm{e}^{+}+\mathrm{e}^{-}\right)$small angle +

$$
+\left(\mathrm{e}^{+}+\mathrm{e}^{-}\right) \text {wide angle }
$$

as discussed in Salvini's talk. On the other hand, a student at Rome, Massimo Rocca, tested this «cascade " approximation in a computation of

$$
\gamma+\gamma \rightarrow \mathrm{e}^{+}+\mathrm{e}^{-}+\mathrm{X},
$$


where $\mathrm{X}$ is a $C=+1$ state $\left(\pi^{0}, \eta\right.$, etc. $)$. The approximation is based on the cascade :

$$
\gamma \rightarrow \mathrm{e}^{+}+\mathrm{e}^{-}, \quad \mathrm{e}^{ \pm} \rightarrow \mathrm{e}^{ \pm}+\gamma, \quad \gamma+\gamma \rightarrow \mathrm{X} .
$$

The leading logarithmic term in the exact calculation is correctly reproduced by the approximate calculation.

P. Kessler. - Yes, such applications are connected with what I called the method of «quasi real processes ", a generalization of the Williams-Weizsäcker method (Nuovo Cimento 17 (1960) 809). We had shown that the $W W$ formula (or equivalent formulae) can be applied to any vertex with one photon and two relativistic charged particles where any one of the three particles is virtual. A former student of mine, Miss Jacquemin, applied the "cascade» technique to the study of the process $\mu \rightarrow y+\bar{v}+\mathrm{e}^{-}+\mathrm{e}^{+}$, occurring through the sequence of « quasi-real processes » :

$$
\mu \rightarrow v+\bar{v}+\mathrm{e}, \quad \mathrm{e} \rightarrow \mathrm{e}+\gamma, \quad \gamma \rightarrow \mathrm{e}^{-}+\mathrm{e}^{+}
$$

(J. Physique 22 (1961) 824). The theoretical result, obtained by computing a double integral (instead of a tenfold integral in the exact calculation), was directly compared with the experimental data of Crittenden et al. (Phys. Rev. 121 (1961) 1823) and the agreement was qualitatively good.

C. Bernardini (Frascati). - Is it understood why the equivalent photon approximation does not work in some apparently simple cases like ee $\rightarrow$ ee $\gamma \gamma$ ? In this reaction, the $\log ^{2}$ term is suppressed.

P. KESSLER. - In double bremsstrahlung, there are many diagrams, and I must confess I am not aware of how the $W W$ approximation was used there. There are indeed some cases of failure of the approximation. These cases occur when the photoproduction process considered contains a physical region close to a pole. Then the virtuality of the quasi-real photon may alter the distance to that pole (even when $q^{2}$ is small) and thus modify the cross section considerably. A detailed discussion on this question, and also an example of such a failure (in the calculation of the reaction $\mu+\mathrm{e} \rightarrow \mu+\mathrm{e}+\mathrm{e}^{-}+\mathrm{e}^{+}$, with muons of $8 \mathrm{GeV}$ incident on an electron target at rest) is given in the second of the two excellent papers of Gorgé et al. (ref. [38] of my talk).

G. Salvini. - What was the contribution of Enrico Fermi to the equivalent photon approximation?

P. KeSSLER. - In the work mentioned (ref. [3] of my talk), Fermi used the classical equivalent photon spectrum in order to evaluate the electro-excitation and electro-ionisation of atoms. He also calculated by this method the energy loss, due to ionisation, of $\alpha$-particles travelling through matter. This latter problem had already been considered by Niels Bohr (ref. [4] of my talk).

L. Stodolsky (Munich). - Did nobody consider an extension of the $W W$ approximation from photonexchange processes to boson exchange (not necessarily vector boson exchange)?

P. KesSLer. - That is another historical point. Such an extrapolation to strong interaction processes was considered by Heitler and Peng (Proc. Roy. Ir. Soc. A 49 (1943) 101 and A 50 (1945) 155), and also by Morpurgo (Nuovo Cimento 6 (1949) 505). I think that these studies may be considered as being at the origin of the so-called peripheral (in particular onepion-exchange) model which was quite popular in the early sixties. But there one must ask : How good is field theory for hadronic processes ? 